\title{
Revisione e sintesi dei modelli teorici del nursing che supportano l'assistenza infermieristica in un contesto multietnico
}

\author{
S. Brenna ${ }^{1}$, A. Oliveira Silva ${ }^{2}$
}

${ }^{1}$ Infermiera volontaria dell'Associazione "Casa dos Sonhos” Santa Rita, Paraiba - Brasile

${ }^{2}$ Coordinatrice del corso di Laurea Magistrale dell’Università Federale del Paraiba, João Pessoa - Brasile

REVIEW AND SYNTHESIS OF THEORETICAL MODELS THAT SUPPORT NURSING IN A MULTIETHNIC CONTEXT

ABSTRACT. Increased needs and chances for every people to move across countries bring nurses to deal with a larger typology of patients coming from different cultures, needs, habits and countries of origin. This phenomena requires nurses to increase their skills in order to provide a quality care to foreigner patients too. In the last 40s years several theoretical models had been produced to support professionals. Main objective of this article is to produce a concise revision of available models, their possible implementation in order to support and help migrated people to benefit of good standard of health.

KEY WoRDs. Theoretical models, Nursing, Trasculturality

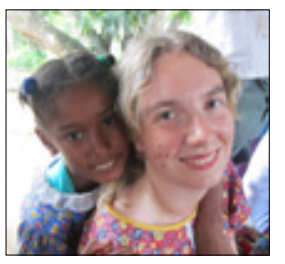

Silvia Brenna

\section{Introduzione}

La complessità dell'attuale contesto sociale porta il professionista sanitario a confrontarsi quotidianamente con un'utenza sempre più varia per cultura, lingua e religione.

Secondo i dati Istat (1), sono $4.570 .317 \mathrm{gli}$ stranieri residenti in Italia al $1^{\circ}$ gennaio 2011,335 mila in più rispetto all'anno precedente $(+7.9 \%)$ : la quota di cittadini stranieri sul totale dei residenti (italiani e stranieri) continua ad aumentare: al $1^{\circ}$ gennaio 2011 è salita al 7.5\% dal 7\% registrato un anno prima.

Attualmente l'Italia, nazione particolarmente esposta per la sua posizione geografica, è diventata uno dei poli preferenziali di immigrazione: il fenomeno migratorio è complesso e vi è un'estrema varietà di soggetti che ne sono protagonisti. Contrariamente a quello che si pensa nella mentalità comune, la popolazione immigrata arriva nel nuovo Paese con un patrimonio di salute pressoché integro, dal momento che, generalmente, sono i soggetti giovani, forti, con più spirito d'iniziativa e maggiore stabilità psicologica che decidono di emigrare.

Tuttavia questo patrimonio è destinato a dissiparsi per le difficoltà che si pongono sul cammino, come gli ostacoli nel trovare un lavoro stabile e ben retribuito e la con- seguente mancanza di reddito, il non riconoscimento di titoli di studio o posizioni professionali acquisite nel proprio Paese, la distanza dalla propria famiglia e dal proprio universo culturale, il cambiamento di clima e di abitudini alimentari e la difficoltà nel reperire un'abitazione degna. Lincontro sempre più frequente nelle nostre realtà ospedaliere e territoriali con persone straniere, introduce una costellazione di nuove variabili nel concetto di salute definita dall'OMS come "stato di completo benessere fisico, psichico e sociale e non semplice assenza di malattia", dove il quadro di completo benessere ci smuove ad affrontare territori a noi estranei, sconosciuti e spesso incomprensibili.

Nella pratica di ogni giorno, questo si traduce in svariate situazioni:

- frequentemente l'utente straniero non si rivolge ai Servizi Sanitari a causa della diversità della lingua, che ancora oggi è uno degli ostacoli maggiori;

- vi è una scarsa conoscenza delle modalità di accesso ai servizi per cui aumenta il ricorso ai servizi di emergenza;

- spesso l'adesione a trattamenti prolungati o cronici è insufficiente, non regolare o interrotta per l'utilizzo di trattamenti naturali e fitoterapici senza il controllo di un professionista;

- le condizioni socio-economiche, i costumi di vita e le 
abitudini alimentari talvolta si trasformano in ostacoli nella cura di alcune patologie croniche;

- pratiche spirituali e religiose incomprensibili per l'operatore, ma di essenziale importanza per la persona, spesso sono causa di serie difficoltà per l'esposizione a pericolo di vita o di contrazione di una malattia sessualmente trasmissibile (ad esempio, infibulazione).

Per non disperderci in questa intricata foresta, possiamo rivolgerci a differenti modelli concettuali che a partire dagli anni Cinquanta sono stati elaborati, in contesti sociali fortemente multiculturali, per aiutare gli operatori sanitari ad orientarsi tra le differenti componenti assistenziali e culturali in relazione tra di loro.

\section{Il "Sunrise Model” o "Modello del Sole Nascente"}

Madeleine Leininger è un'infermiera antropologa originaria dello Stato del Nebraska (USA). La Leininger si rese conto delle differenze culturali dei bambini che afferivano al Servizio di Psichiatria in cui lavorava e della mancanza delle competenze necessarie, da parte di tutte le figure sanitarie, per affrontare tale complessità. Per questo nel 1961 elaborò la teoria delle diversità e universalità dell'assistenza transculturale, definendo il nursing transculturale come "un'area principale dell'assistenza che ha al suo centro uno studio comparato con l'analisi delle diverse culture e subculture mondiali in riferimento al loro comportamento di assistenza nei confronti dei malati, dei valori sanitari di salute e malattia; teorie e modelli di comportamento con l'obiettivo di sviluppare un corpo di conoscenze per fornire indicazioni di assistenza sia di specifiche culture che universali" (2) Il "Modello del Sole Nascente", chiamato così per la particolare rappresentazione grafica (Fig. 1), descrive quali sono i quattro livelli di influenza sulla salute e sull'assistenza degli individui nelle varie culture del mondo:

- visione del mondo e sistema sociale;

- conoscenze correlate allindividuo, alla famiglia, ai gruppi e alle istituzioni nei diversi sistemi sanitari;

- il sistema popolare che riguarda le credenze legate alla malattia, alla salute e alla guarigione;

- le decisioni e le azioni assistenziali che possono essere di tre tipi:

a) conservazione/mantenimento, cioè quelle azioni che aiutano la persona a mantenere uno stato di salute, a guarire da una malattia o a prepararsi per la morte;

b) negoziazione/adattamento, cioè quelle azioni che portano la persona ad adattarsi ad una condizione o a negoziare una situazione di salute più soddisfacente;

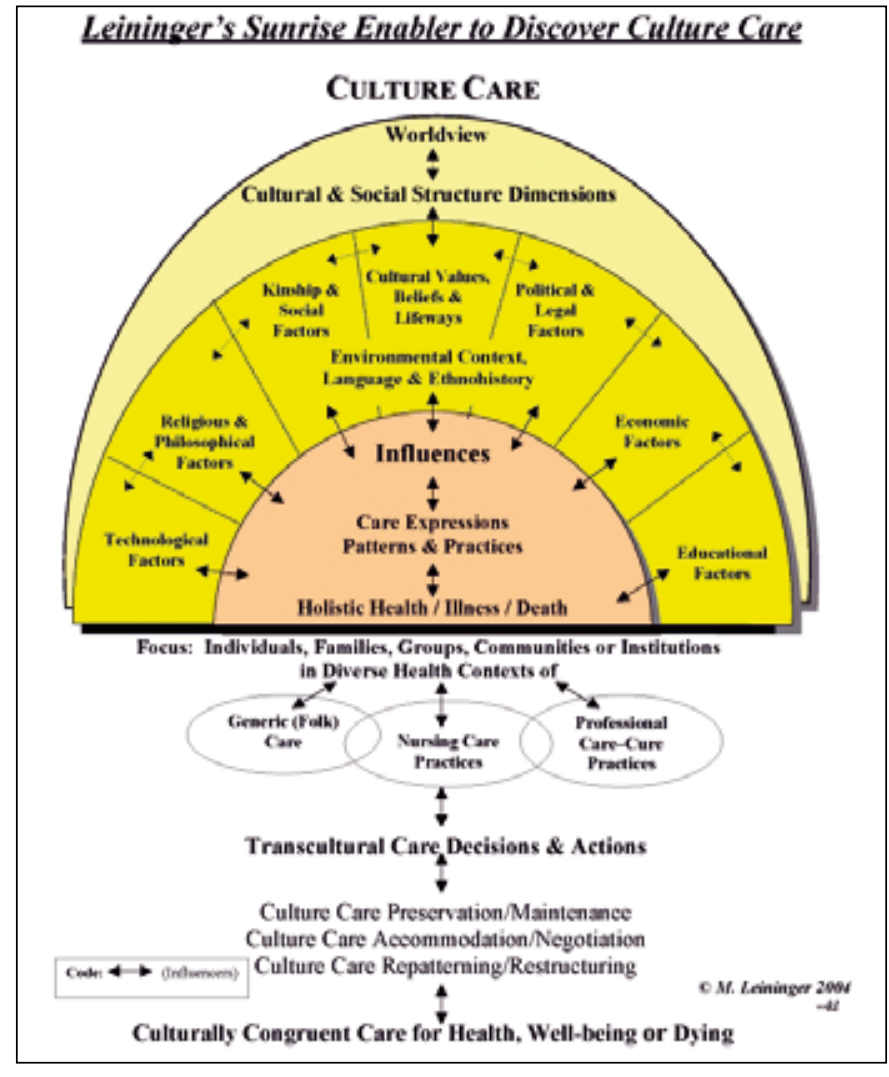

Fig. 1 - Rappresentazione grafica del modello del Sole Nascente.

c) rimodellamento/rielaborazione, cioè quelle azioni che aiutano il paziente a cambiare i propri stili di vita.

Madeleine Leininger sostiene che l'obiettivo di questo modello è arrivare a una comprensione dell'assistenza e del concetto di salute nelle diverse culture, così da poter rendere le persone capaci di trovare le risposte più adeguate per raggiungere gli obiettivi di salute.

\section{Il modello delle competenze professionali e dell'ascolto}

Secondo il modello di Papadopoulos, Tilki e Taylor, pubblicato per la prima volta nel 1998, le competenze culturali e le abilità di ascolto possono essere competenze complementari per gli operatori sanitari nell'assistenza interculturale.

Il modello si sviluppa in quattro fasi che interagiscono tra di loro:

\section{Consapevolezza culturale}

- Autoconsapevolezza

- Identità culturale 


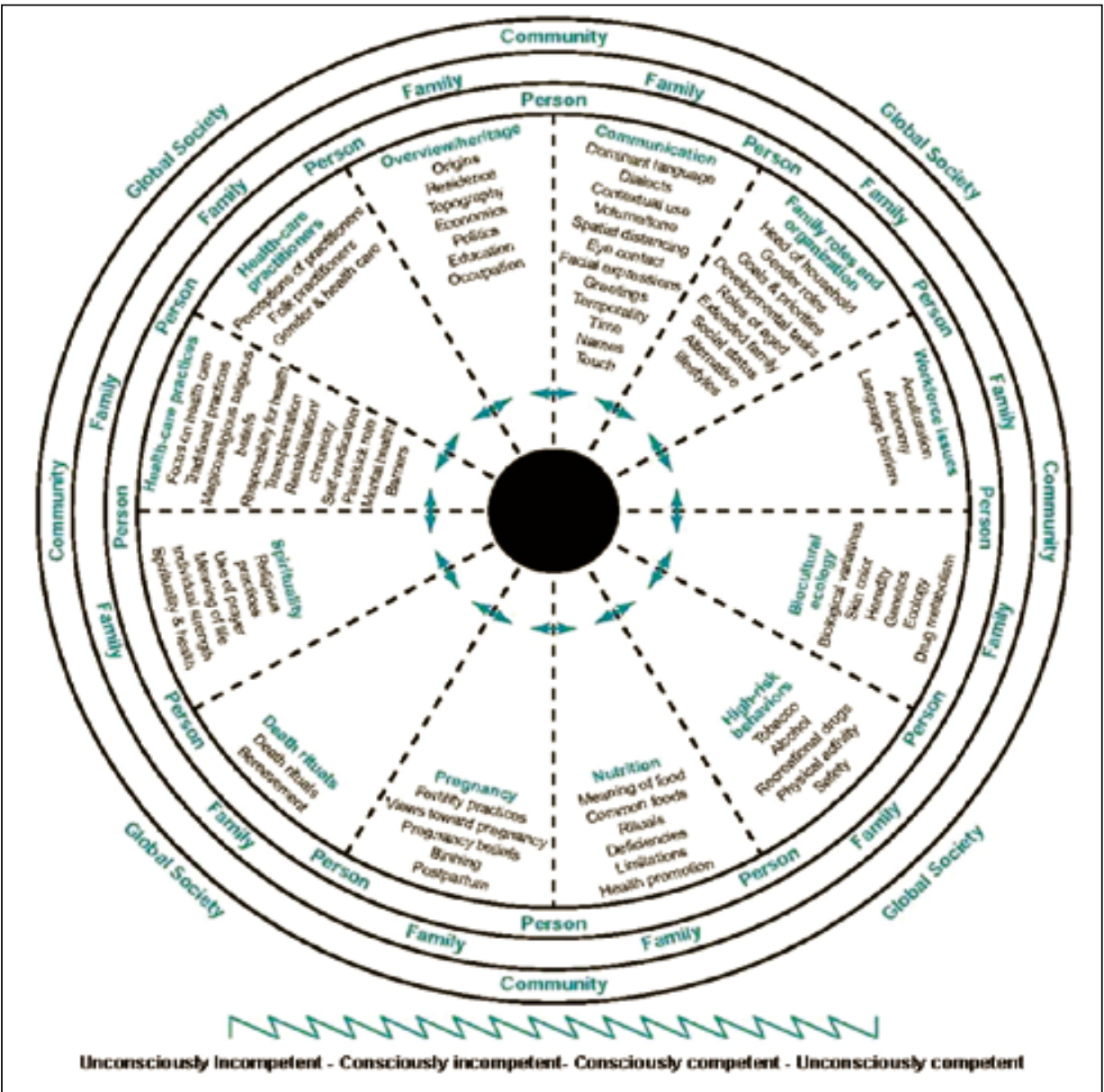

Fig. 2 - Rappresentazione grafica del modello di Purnell e Paulanka.

- Attaccamento all'eredità e al patrimonio familiare

- Etnocentrismo

\section{Conoscenza culturale}

- Credenze e comportamenti sulla salute

- Barriera alla sensibilità culturale

- Stereotipo

- Etnostoria

- Comprensione sociologica

- Somiglianze e diversità

\section{Sensibilità culturale}

- Empatia

- Capacità comunicative e interpersonali

- Fiducia

- Accoglienza

- Appropriatezza

- Rispetto

\section{Competenza culturale}

- Capacità di valutazione

- Capacità di diagnosi
- Capacità cliniche

- Confronto con il pregiudizio, la discriminazione e le disuguaglianze

Questo modello (3) si basa prima di tutto sulla presa di coscienza della propria identità culturale, quindi sulle capacità personali del professionista come il rispetto della diversità, la flessibilità, la capacità di ascolto e di dialogo.

\section{Il modello olografico di Purnell e Paulanka}

Professori di Nursing all'Università di Delegare, Purnell e Paulanka elaborarono questo modello (4) nel 1998 negli Stati Uniti. Il modello è graficamente rappresentato con un cerchio (Fig. 2) il cui bordo esterno rappresenta la società globale (comunicazione, politica, conflitti, disastri naturali, scambi commerciali ed educativi, progressi della scienza, opportunità di viaggiare e di interagire con altri individui).

Il secondo bordo rappresenta la comunità, come gruppo di persone accomunate da un interesse e un'identità (ca- 
ratteristiche sociali, fisiche e simboliche come la religione, la lingua, gli stili di vita, la storia).

Il terzo bordo rappresenta la famiglia, ovvero il legame che unisce due persone senza relazioni di parentela, ma unite affettivamente. Influenzano la struttura familiare diversi fattori come l'età, la generazione, lo stato socio-economico. Il quarto circolo rappresenta la persona, un essere biopsico-socio-culturale dinamico, in continuo adattamento con la sua comunità: gli esseri umani si adattano dinamicamente ai processi di cambiamento in accordo con la famiglia, la comunità e la più ampia società globale.

Linterno del cerchio è diviso in 12 spicchi che rappresentano i diversi significati di cultura correlati tra loro (visione del mondo e topografia, comunicazione, ruoli familiari, concetti culturali legati al lavoro, ecologia, comportamenti a rischio, nutrizione, gravidanza e pratiche legate al concepimento, rituali, spiritualità, pratiche di salute ed assistenza, coloro che eseguono le pratiche assistenziali). Al centro il cerchio nero indica i fenomeni sconosciuti della cultura di riferimento.

In basso al cerchio c'è una linea discontinua che riguarda il percorso dell'operatore e dei sistemi sanitari, che percorrono 4 tappe: incompetenza inconscia, incompetenza conscia, competenza conscia, competenza inconscia.

\section{Il modello di Campinha-Bacote}

La teorica Campinha-Bacote nel 1991 identifica la competenza culturale come un processo dinamico (5), in cui l'operatore sanitario rinnova continuamente le sue abilità per aumentare l'efficacia del suo lavoro all'interno dell'universo culturale dell'utente. É un modello di apprendimento continuo da parte dell'infermiere composto da quattro concetti e cinque assunti, relazionati tra di loro, che l'operatore deve sviluppare per avere un'adeguata competenza nel nursing:

- la propria consapevolezza culturale

- la conoscenza culturale dell'altro

- l'abilità culturale

- l'incontro culturale

- il desiderio culturale, che è l'energia che spinge l'infermiere verso la competenza culturale.

\section{Il modello delle fasi di valutazione}

Giger e Davidhizar (6) nel 1991 descrissero sei fenomeni culturali che l'operatore sanitario deve comprendere per fornire cure efficaci a tutti i pazienti:

- Comunicazione, che riguarda i problemi di linguaggio e le incomprensioni.

- Spazio, riferito alla percezione dello spazio, la prossi- mità agli altri, i movimenti corporei.

- Organizzazione sociale, che comprende la posizione geografica, religione, stato socio-economico, orientazione sessuale.

- Tempo, nel concetto di passaggio del tempo e durata.

- Controllo dell'ambiente, che riguarda l'abilità delle persone di controllare la natura.

- Variazione biologica, che si riferisce alla struttura del corpo correlato, al colore della pelle, alla consistenza dei capelli e altre caratteristiche fisiche, alle variazioni enzimatiche e genetiche.

Coerentemente con i fenomeni culturali di cui sopra, Boyle e Andrews (1989) (7) sostennero che gli infermieri devono valutare otto aree che riflettono differenze culturali e raccogliere i seguenti dati:

- Storia delle origini della cultura dei pazienti.

- Orientamenti dei principi, tra cui visione del mondo, l'etica e le norme e gli standard di comportamento sul tempo, lavoro, denaro, educazione, bellezza, forza, e cambiamento.

- Relazioni interpersonali, compresi i modelli familiari, comportamento, ruoli e relazioni.

- Modelli di comunicazione.

- Religione e magia.

- Sistemi sociali, inclusi i valori economici, sistemi politici e modelli educativi.

- Dieta e abitudini alimentari.

- Sistemi di credenze sulla salute e sulla malattia.

\section{Discussione}

I modelli citati possono essere divisi in due classi: quelli creati per essere applicati dal professionista e quelli creati per l'insegnamento e la creazione di competenze nel professionista.

Il modello della Leininger è, oltre che il più antico, il principale tra quelli nati per essere strumento nelle mani del professionista. Non a caso il primo studio che Madeleine Leininger portò a termine fu "sul campo", osservando la tribù dei Gadsup in Nuova Guinea (8).

Il centro della teoria della Leininger è proprio lo studio della persona, delle sue esperienze e conoscenze locali, del suo concetto di salute e del concetto di assistenza, come insieme delle azioni che, secondo l'individuo, portano alla salute.

11 modello delle fasi di valutazione si rifà al modello della Leininger, sottolineando l'importanza della raccolta dati da parte dellinfermiere per avere una descrizione dettagliata del mondo del paziente e fornire le cure adeguate.

Il modello di Campinha, dei primi anni Novanta, fu un ponte verso il cambiamento di ottica: tale modello infatti sottolinea l'importanza di un processo dinamico di apprendimen- 
to dell'infermiere per aumentare le proprie competenze nell'entrare nell'universo dell'altro. Tutto ciò, però, è ancora lasciato alla responsabilità e alla pratica del professionista.

Il modello di Papadopoulos si propose come guida per la strutturazione di moduli didattici, corsi di perfezionamento o progetti di ricerca applicata alla pratica assistenziale.

Il modello di Purnell nacque invece come strumento di valutazione clinica di studenti infermieri per poi essere utilizzato per conoscere le caratteristiche culturali dei pazienti e delle loro famiglie. In seguito vennero anche introdotte scale di valutazione delle competenze culturali dei professionisti.

La particolarità di questo modello è che può essere applicato in un sistema multidisciplinare tra vari professionisti per uniformare l'assistenza ai pazienti, caratteristica che si inserisce alla perfezione in un sistema di case management.

\section{Conclusioni}

Il professionista sanitario dovrà sempre più affrontare la sfida di assistere un'utenza ogni giorno più "varia e colorata". Per questo si rende necessario una nuova forma mentale nella quale l'adozione di un modello teorico può aiutare a comprendere la persona che ci sta di fronte e a uniformare la pianificazione degli interventi con l'équipe multidisciplinare.

Accanto a questa necessità, a parere dell'autore, è fondamentale giocare le proprie competenze personali nel percepire la transculturalità non come il tentativo di creare un insieme di esseri simili, ma nell'apprezzare la diversità come una ricchezza attraverso la quale l'infermiere può acquisire nuove possibilità per incrementare le proprie capacità e abilità come persona e come professionista sanitario.

Certo nel campo sanitario gli ostacoli sono molti e non solo culturali e religiosi, ma soprattutto socio-economici, politici, amministrativi e organizzativi, ostacoli che spesso affondano la "buona volontà".

Per questo il cominciare a pensare seriamente di costruire nelle nostre unità operative dei progetti con delle fondamenta importanti, come dei modelli teorici di riferimento, che non siano legati solo alle capacità e alla volontà del singolo, ma piuttosto inserire il nursing transculturale nella formazione di base o nell'educazione continua, si rende sempre più necessario per offrire all'utenza una assistenza personalizzata e di qualità. «Linfermiere presta assistenza secondo principi di equità e giustizia, tenendo conto dei valori etici, religiosi e culturali, nonché del genere e delle condizioni sociali della persona.»

«L'infermiere orienta la sua azione al bene dell'assistito di cui attiva le risorse sostenendolo nel raggiungimento della maggiore autonomia possibile, in particolare, quando vi sia disabilità, svantaggio, fragilità.»

\section{(Articoli 4 e 7 del Codice Deontologico)}

\author{
Indirizzo degli Autori: \\ Silvia Brenna \\ Rua Joaquim Gomes De Castro, 488 \\ Loteamento Boa Vista \\ 58.300-970 Santa Rita-PB, Brasil \\ silvia.brenna@alice.it \\ www.casadosonhos.org
}

\section{Bibliografia}

1. www.istat.it

2. Leininger MM. Culture Care Diversity and Universality. A Worldwide Nursing Theory, Sudbury MA, Jones \& Barlett Publischers, 2001.

3. Papadopoulos I. Transcultural Health and Social Care. Edimburgo: Churchill Livingstone Elsevier, 2006.

4. Purnell L. The Purnell model for cultural competence. J Transcult Nurs 2002; 13(3): 193-6.

5. Campinha-Bacote J. Coming to Know Cultural Competence: An Evolutionary Process. IJHC 2011; 15(3): 42-8.

6. Giger JN, Davidhizar RE. Transcultural Nursing: Assessment and Intervention. St. Louis: CV Mosby, 1991.

7. Andrews MM, Boyle JS. Transcultural concepts in nursing care. J Transcult Nurs 2002; 13(3): 178-80.

8. Leiniger MM. Gadsup of Papua New Guinea Revisited: A Three Decade View. J Transcult Nurs Summer 1993; 5 (1): 21-30.

9. www.transculturalcare.net

10. Bertolini R, Bombardi S, Manfreini M. Assistenza Infermieristica e Ostetrica. Teoria -Metodologia- Applicazioni. Edizione Hoepli 2009. 